\title{
Acceso minipterional interfascial simple y combinado, con peeling de fosa media y clinoidectomia anterior extradural. Serie clínica Simple and combined interfascial minipterional approach, with medium fosa peeling and anterior extradural clinoidectomy. Clinical series
}

Iván Perales Cabezas ${ }^{1}$

1 Neurocirujano Vascular y de la Base del Cráneo. Hospital San Pablo y Clínica Elqui. Región de Coquimbo, Chile.

Rev. Chil. Neurocirugía 45: 122-130, 2019

\section{Resumen}

Introducción: En las últimas dos décadas al acceso pterional clásico descrito por Yasargil se le han realizado modificaciones importantes en búsqueda de acceder a la base del cráneo, tales como el acceso transcigomático y orbitocigomático; más recientemente, accesos mínimamente invasivos, abordajes keyhole. Es así como se ha descrito el acceso minipterional con disección interfascial, el que ha sido combinado con técnicas de la base del cráneo, clinoidectomía anterior extradural, peeling de fosa media y petrosectomía anterior de Kawase, por el equipo de Neurocirugía Vascular y de la base del Cráneo del Instituto de Neurocirugía (INCA), a cargo del Dr. Jorge Mura, logrando resolver patologías complejas por un acceso keyhole. Este trabajo presenta una serie clínica, multicéntrica, realizada por un mismo neurocirujano, y que tiene como objetivo demostrar que el acceso minipterional simple y combinado es reproducible en otros centros, con buenos resultados clínicos. Materiales y Métodos: El autor presenta una serie clínica, de 56 pacientes, tratados en el hospital San Pablo de Coquimbo y Clínica Elqui de La Serena, entre abril de 2017 y agosto de 2018, con un seguimiento prospectivo de los resultados clínicos, los cuales fueron evaluados con la escala de Rankin modificado. Resultados: En un total de 56 casos, predominó el sexo femenino (71\%), la edad media fue de 53 años y la patología aneurismática fue lo más frecuente con 45 casos, de estos el $60 \%$ fueron aneurismas rotos; el $7 \%$ correspondieron a tumores de la base del cráneo y el $12,5 \%$ a hidrocefalias no comunicantes resueltas por la apertura de la lámina terminalis. En 9 casos se combinó el acceso minipterional con clinoidectomía anterior extradural y en 3 casos se hizo un peeling de fosa media. El mayor tiempo quirúrgico estuvo asociado al acceso minipterional combinado, ya sea por patología tumoral o aneurismas paraclinoideos. Se obtuvo un resultado neurológico favorable (ERm < 3) en el $87,5 \%$ de los pacientes. Conclusión y Discusión: actualmente la evidencia ha dejado de manifiesto que el acceso minipterional tiene ventajas sobre otros accesos keyhole, principalmente por la posibilidad de poder combinarlo con técnicas de base del cráneo, pudiendo resolver patologías que antes no era posible tratar por accesos mínimamente invasivos. El acceso pterional clásico, sin embargo, sigue teniendo indicación en casos seleccionados. Por otra parte, esta serie clínica confirma que el acceso minipterional simple y combinado, es reproducible y que, utilizándolo con un adecuado manejo microquirúrgico, logra buenos resultados en patologías complejas.

Palabras clave: Craneotomía minipterional, aneurisma, meningioma, mínimamente invasiva.

\begin{abstract}
Introduction: In the last two decades, the classic pterional approach described by Yasargil has undergone important modifications in search of access to the base of the skull, such as transcigomatic and orbitozygomatic approach; more recently, minimally invasive accesses, keyhole approaches. This is how minipterional access with interfascial dissection has been described, which has been combined with techniques of the base of the skull, anterior extradural clinoidectomy, middle fossa peeling and Kawase anterior petrosectomy, by the Vascular Neurosurgery team and the base of the Skull of the Institute of
\end{abstract}


Neurosurgery (INCA), by Dr. Jorge Mura, managing to solve complex pathologies through a keyhole access. This work presents a clinical, multicentric series, carried out by the same neurosurgeon, and whose objective is to demonstrate that simple and combined mini-pterional access is reproducible in other centers, with good clinical results. Materials and Methods: The author presents a clinical series of 56 patients, treated at the San Pablo of Coquimbo hospital and the Elqui clinic of La Serena, between April 2017 and August 2018, with a prospective follow-up of the clinical results, which were evaluated with the modified Rankin scale. Results: In a total of 56 cases, the female sex predominated (71\%), the average age was 53 years and the aneurysmal pathology was the most frequent with 45 cases, of these $60 \%$ were ruptured aneurysms; $7 \%$ corresponded to tumors of the base of the skull and $12.5 \%$ to non-communicating hydrocephalus resolved by the opening of the lamina terminalis. In 9 cases, mini-pterional access was combined with extradural anterior clinoidectomy and in 3 cases a middle fossa peeling was performed. The greater surgical time was associated with combined mini-pterional access, either due to tumoral pathology or paraclinoid aneurysms. A favorable neurological result $(E R m<3)$ was obtained in $87.5 \%$ of the patients. Conclusion and Discussion: Currently the evidence has shown that the minipterional approach has advantages over other keyhole approaches, mainly due to the possibility of being able to combine it with skull base techniques, being able to resolve pathologies that previously could not be treated by minimally invasive approaches. The classical pterional approach, however, continues to be indicated in selected cases. On the other hand, this clinical series confirms that simple and combined minipterional approach is reproducible and that, using it with adequate microsurgical management, it achieves good results in complex pathologies.

Key works: Minipterional craniotomy, aneurysms, meningioma, minimally invasive.

\section{Introducción}

El acceso pterional o frontotemporal, descrito por Gazi Yasargil en el año 1975 para acceder al piso anterior, medio, aneurismas de circulación anterior y arteria basilar distal, tumores de la región selar, paraselar, quiasmática y subfrontal ${ }^{1,2}$, ampliamente utilizado por los neurocirujanos, ha tenido variaciones que han sido descritas por diferentes autores, como el acceso transcigomático y orbitocigomático ${ }^{3}$, en búsqueda de una mejor exposición de las estructuras neurovasculares y piso de fosa media y/o seno cavernoso, sin embargo, estos accesos incluido el pterional clásico, tienen desventajas tales como retracción de los tejidos blandos, problemas de cicatrización de la piel en línea media, atrofia del músculo temporal, lesión incidental de arteria temporal superficial ${ }^{4}$, dolor al masticar durante el periodo post operatorio, craneotomía amplia y en ocasiones exposición del seno frontal, teniendo complicaciones como la fístula de líquido cefalorraquí$\mathrm{deo}^{12}$. Por estas razones, otros autores han realizado modificaciones del acceso pterional, buscando evitar estas complicaciones además de disminuir el tiempo quirúrgico y obtener mejores resultados estéticos, sin perjuicio de las ventajas que ofrece el acceso pterional clásico ${ }^{5,6}$. Nathal en el 2005 describe el acceso minipterional miocutáneo ${ }^{7}$ y Figueiredo en el 2007 describe el minipterional con disección interfascial ${ }^{8}$, ambos teniendo como característica principal, realizar la craneotomía no más allá de la línea temporal superior, teniendo variantes en su extensión antero-posterior según sea necesario. El Dr. Jorge Mura, combinó este acceso con clinoidectomia anterior extradural, descrita por Hakuba ${ }^{8}$ y popularizada por Dolenc ${ }^{10,11}$, peeling de fosa media y petrosectomía anterior de Kawase, pudiendo resolver patologías vasculares y tumorales de ubicaciones que antes se consideraban inaccesibles por esta vía, obteniendo el primer lugar en el congreso chileno de neurocirugía del año 2016. El autor de esta serie, adquirió el conocimiento de este acceso, minipterional simple y combinado, en su estadía en el fellow de Neurocirugía Vascular y Base del Cráneo, en el Instituto de Neurocirugía Asenjo (INCA), durante el período comprendido entre abril de 2016 y abril de 2017, pudiendo posteriormente, desarrollar este acceso en el hospital San Pablo de Coquimbo y clínica Elqui.

En esta serie clínica personal, se describen patologías complejas tanto vasculares, como tumorales e hidrocefalias no comunicantes, resueltas por el acceso minipterional simple o combinado. Teniendo como objetivo general caracterizar a los pacientes y describir las patologías que fueron resueltas por esta vía y como objetivos específicos analizar los tiempos quirúrgicos y los resultados funcionales de los pacientes.

\section{Materiales y Métodos}

Se presenta una serie clínica, de diversas patologías neuroquirúrgicas complejas, vasculares, tumorales que compromete la fosa media, la región paraclinoidea y seno cavernoso, así como también hidrocefalias no comunicantes, tratadas con apertura de la lámina terminalis. Todas abordadas por el acceso minipterional, con disección interfascial del musculo temporal, y según corresponda, combinado con peeling de fosa media y clinoidectomia anterior extradural. Todos los pacientes fueron tratados por el autor de este trabajo, en el hospital clínico San Pablo de Coquimbo y Clínica Elqui de La Serena, en un período de 17 meses, desde abril de 2017 hasta agosto de 2018, con un total de 56 casos. El control y seguimiento de los pacientes, fue realizado por el mismo autor.

Durante el período que comprendió esta serie clínica, solo se operó una paciente por vía pterional clásica, que no se incluye en los resultados, un meningioma del tubérculo selar, que se extendía hacia lateral hasta ambas bifurcaciones carotideas y hacía posterior hasta la cisterna interpeduncular, por lo que el autor planificó la resección tumoral a través del valle silviano y por subfrontal no solo desde lateral, lo que ofrece el minipterional, sino también desde anterior, lo que ofrece la craneotomía pterional clásica.

Se realizó un análisis prospectivo de 
los resultados neurológicos funcionales, con un seguimiento de hasta 16 meses. Se definió un resultado neurológico favorable para aquellos pacientes con una escala de Rankin modificado $(\mathrm{ERm})$ menor a 3 y un resultado neurológico no favorable para aquellos pacientes con una ERm mayor o igual a 3. Además, se describe la condición pre operatoria, el tiempo quirúrgico, las complicaciones y los resultados estéticos.

\section{Descripción de la técnica quirúrgica}

La posición del paciente en decúbito supino, mínima tricotomía, con la cabeza en línea media deflectada, excepto en el acceso minipterional combinado donde la cabeza necesita estar rotada 30-40 grados para realizar la clinoidectomía o el peeling de fosa media en forma más cómoda. Se utilizó un catéter espinal en los pacientes que se realizó un peeling de fosa media. Se realiza una incisión arciforme siguiendo la línea de inserción del pelo, de $8 \mathrm{~cm}$ de longitud (Figura 1), llegando a la altura de la línea media pupilar, sin llegar a la línea media (Figura 2), un colgajo interfascial, preservando la arteria temporal superficial y la rama frontal del nervio facial (Figura 3). La desinserción del músculo temporal se realizó con monopolar (Figura 4), una craneotomía variable en tamaño según la patología a tratar, en promedio $3 \times 3 \mathrm{~cm}$, teniendo como requisito fundamental no traspasar la línea temporal superior (Figura 5), la durotomía fue arciforme en todos los casos, excepto en los combinados con peeling de fosa media, donde se trabajó por extradural.

\section{Resultados}

Una serie clínica de un total 56 casos, operados por vía minipterional. Con un rango de edad entre los 16 y los 78 años, con un promedio de 53 años, el sexo femenino fue predominante con el $71 \%$. Veintisiete casos de hemorragia subaracnoidea aneurismática, 18 casos de aneurismas no rotos, 6 aneurismas paraclinoideos, 1 caso de aneurisma de circulación posterior, 7 casos de hidrocefalia no comunicante, con apertura de la lámina terminalis y 4 casos de tumores de la base de cráneo (Tablas 1, 2, 3 y grafico 1).

En 45 pacientes se realizó una craneotomía minipterional simple (Tablas 1.1,

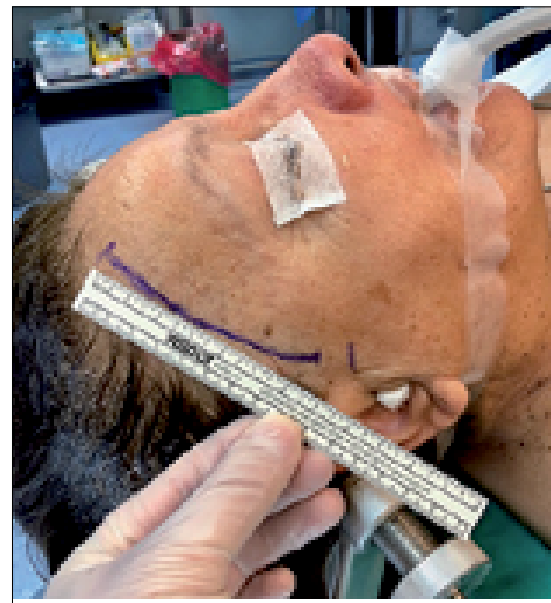

Figura 1. Marca de la incisión, 7-8 cm de longuitud. La cabeza posicionada sin rotación y deflectada.

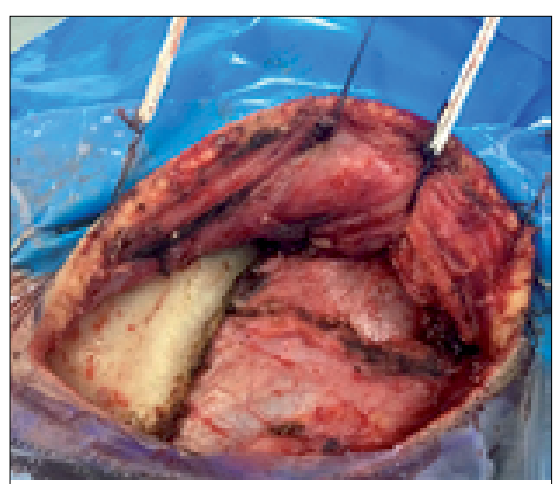

Figura 3. Disección interfascial y desinserción del músculo temporal de la linea temporal superior.

1.2 y 1.3); en 11 casos se realizó un acceso minipterional combinado, 8 de estos fue combinado con clinoidectomía extradural, en dos casos se combinó con peeling de fosa media y en un caso se realizó clinoidectomia más peeling de fosa media (Tabla 2).

De un total de 27 casos de HSA, 23 pacientes fueron tratados por acceso minipterional simple y 4 combinados con clinoidectomia extradural. De los $18 \mathrm{ca}-$ sos de aneurismas no rotos, 15 fueron resueltos por la vía minipterional simple y 3 combinado con clinoidectomia, en un solo caso de aneurisma paraclinoideo, carótido-oftálmico no roto, no fue necesario realizar la clinoidectomia extradural.

De los 4 casos de tumores de la base del cráneo, todos se resolvieron por acceso minipterional combinado, 2 con peeling de fosa media, uno con clinoidectomia extradural y un caso con pee-

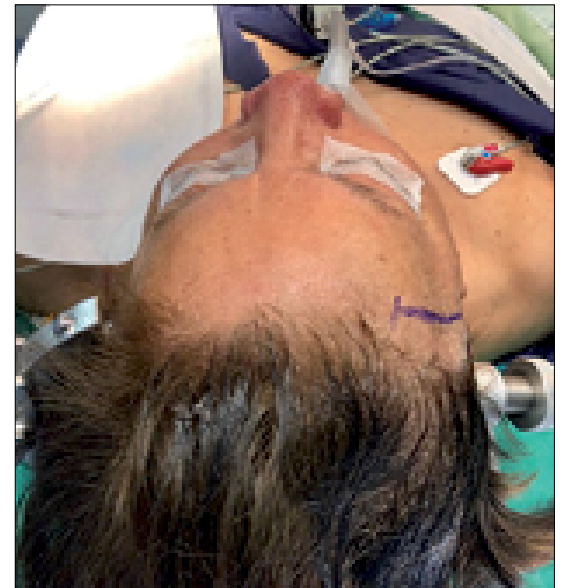

Figura 2. La incisión llega a la altura de la línea media pupilar.

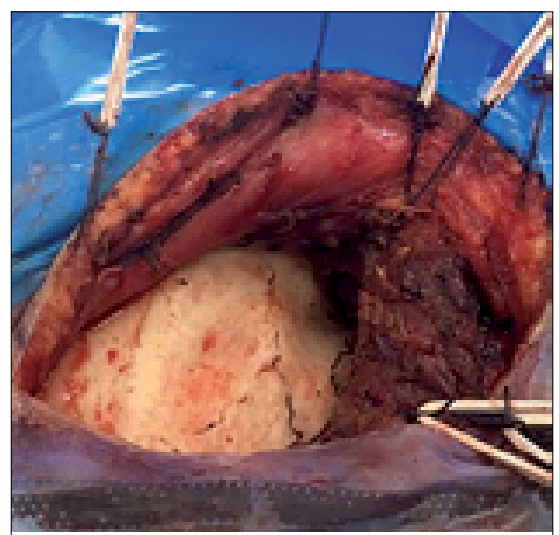

Figura 4. Se refleja el músculo temporal, exponiendo el pterion y la escama temporal.

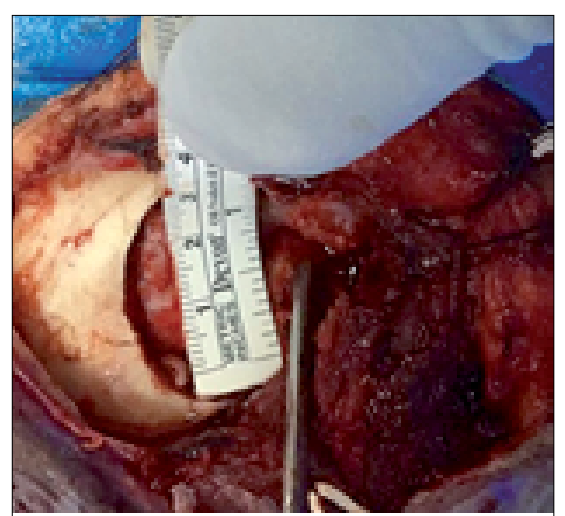

Figura 5. Craneotomía minipterional simple, en un paciente con aneurisma silviano roto.

ling más clinoidectomia.

El tiempo quirúrgico promedio más alto fue en relación a los tumores de base 
Tabla 1.1.

Pacientes con diagnóstico de HSA por aneurisma roto, operados por minipterional simple

\begin{tabular}{|c|c|c|c|}
\hline Fecha & Edad & Diagnóstico & Tiempo quirúrgico \\
\hline 03/04/2017 & 67 & HSA an silviano derecho & $4 \mathrm{~h} 34 \mathrm{~min}$ \\
\hline 02/05/2017 & 41 & HSA an silviano derecho & 4 h $52 \min$ \\
\hline $04 / 05 / 2017$ & 70 & HSA an acop gigante roto & $5 \mathrm{~h} 13 \mathrm{~min}$ \\
\hline $14 / 05 / 2017$ & 42 & HSA an acoa roto & $4 \mathrm{~h} 56 \mathrm{~min}$ \\
\hline $24 / 05 / 2017$ & 53 & HSA an acop roto & $4 \mathrm{~h} 47 \mathrm{~min}$ \\
\hline 20/06/2017 & 63 & HSA an acop roto & $6 \mathrm{~h} 05 \mathrm{~min}$ \\
\hline $11 / 07 / 2017$ & 57 & HSA an acop roto & $4 \mathrm{~h} 23 \mathrm{~min}$ \\
\hline $13 / 07 / 2017$ & 53 & HSA an acop roto & $3 \mathrm{~h} 55 \mathrm{~min}$ \\
\hline $13 / 08 / 2017$ & 52 & HSA an acoa roto & $6 \mathrm{~h} 04 \mathrm{~min}$ \\
\hline 08/10/2017 & 44 & HSA an acoa roto & $4 \mathrm{~h} 57 \mathrm{~min}$ \\
\hline $15 / 11 / 2017$ & 69 & HSA an acoa roto & $5 \mathrm{~h} 24 \mathrm{~min}$ \\
\hline 05/12/2017 & 58 & HSA an acoa roto & $4 \mathrm{~h} 06 \mathrm{~min}$ \\
\hline $17 / 12 / 2017$ & 65 & HSA an acop roto & $4 \mathrm{~h} 07 \mathrm{~min}$ \\
\hline 23/12/2017 & 67 & HSA an acoa roto & $4 \mathrm{~h} 22 \mathrm{~min}$ \\
\hline 02/01/2018 & 78 & HSA an acop roto & $3 \mathrm{~h} 18 \mathrm{~min}$ \\
\hline 03/01/2018 & 64 & HSA an silviano roto & $4 \mathrm{~h} 20 \mathrm{~min}$ \\
\hline $15 / 01 / 2018$ & 75 & HSA an silviano roto & 5 h 57 min \\
\hline $18 / 01 / 2018$ & 29 & HSA an acoa roto & $4 \mathrm{~h} 30 \mathrm{~min}$ \\
\hline $19 / 02 / 2018$ & 44 & HSA an silviano roto & $5 \mathrm{~h} 15 \mathrm{~min}$ \\
\hline $12 / 06 / 2018$ & 58 & HSA bifurcación carotidea roto & $3 \mathrm{~h} 47 \mathrm{~min}$ \\
\hline $17 / 06 / 2018$ & 53 & HSA an acoa roto & $4 \mathrm{~h} 27 \mathrm{~min}$ \\
\hline 07/07/2018 & 39 & HSA an silviano roto & $5 \mathrm{~h} 50 \mathrm{~min}$ \\
\hline $31 / 07 / 2018$ & 78 & HSA an acoa roto & $3 \mathrm{~h} 50 \mathrm{~min}$ \\
\hline
\end{tabular}

\section{Tabla 1.2.}

Pacientes con diagnóstico de aneurisma cerebral no roto. Operados por minipterional simple

\begin{tabular}{|l|l|l|c|}
\hline Fecha & Edad & \multicolumn{1}{|c|}{ Diagnóstico } & Tiempo quirúrgico \\
\hline $04 / 05 / 2017$ & 60 & an acoa no roto & $4 \mathrm{~h} 05 \mathrm{~min}$ \\
\hline $15 / 05 / 2017$ & 60 & an silviano no roto & $4 \mathrm{~h} 34 \mathrm{~min}$ \\
\hline $22 / 05 / 2017$ & 61 & an acop no roto & $3 \mathrm{~h} 46 \mathrm{~min}$ \\
\hline $20 / 08 / 2017$ & 67 & an coroideo no roto & $4 \mathrm{~h} 00 \mathrm{~min}$ \\
\hline $01 / 09 / 2017$ & 68 & an silviano no roto & $4 \mathrm{~h} 38 \mathrm{~min}$ \\
\hline $14 / 09 / 2017$ & 67 & an paraclinoideo no roto & $4 \mathrm{~h} 15 \mathrm{~min}$ \\
\hline $10 / 02 / 2018$ & 60 & an acop sintomático & $4 \mathrm{~h} 49 \mathrm{~min}$ \\
\hline $15 / 03 / 2018$ & 67 & an silviano no roto & $4 \mathrm{~h} 18 \mathrm{~min}$ \\
\hline $10 / 06 / 2018$ & 30 & an acop sintomático & $4 \mathrm{~h} 30 \mathrm{~min}$ \\
\hline $14 / 06 / 2018$ & 49 & an bifurcación carotidea no roto & $3 \mathrm{~h} 30 \mathrm{~min}$ \\
\hline $22 / 06 / 2018$ & 49 & an silviano no roto & $4 \mathrm{~h} 00 \mathrm{~min}$ \\
\hline $16 / 07 / 2018$ & 45 & an arteria cerebral media no roto & $4 \mathrm{~h} 15 \mathrm{~min}$ \\
\hline $23 / 08 / 2018$ & 63 & an silviano derecho no roto & $4 \mathrm{~h} 06 \mathrm{~min}$ \\
\hline $23 / 08 / 2018$ & 65 & an silviano derecho no roto & $3 \mathrm{~h} 55 \mathrm{~min}$ \\
\hline $30 / 08 / 2018$ & 64 & an acop no roto izquierdo & $3 \mathrm{~h} 10 \mathrm{~min}$ \\
\hline
\end{tabular}


Tabla 1.3.

Pacientes con diagnóstico de hidrocefalia no comunicante, operados por minipterional simple

\begin{tabular}{|l|c|l|c|}
\hline Fecha & Edad & \multicolumn{1}{c|}{ Diagnóstico } & Tiempo quirúrgico \\
\hline $17 / 10 / 2017$ & 55 & Estenosis acueductal & $2 \mathrm{~h} 10 \mathrm{~min}$ \\
\hline $27 / 11 / 2017$ & 70 & Estenosis acueductal & $2 \mathrm{~h} 35 \mathrm{~min}$ \\
\hline $28 / 11 / 2017$ & 16 & Tumor epidermoide fosa posterior & $1 \mathrm{~h} 53 \mathrm{~min}$ \\
\hline $14 / 12 / 2017$ & 38 & Estenosis acueductal & $1 \mathrm{~h} 34 \mathrm{~min}$ \\
\hline $12 / 06 / 2018$ & 20 & Tumor epidermoide del acueducto & $1 \mathrm{~h} 55 \mathrm{~min}$ \\
\hline $11 / 07 / 2018$ & 60 & Glioma/obstrucción acueductal & $1 \mathrm{~h} 43 \mathrm{~min}$ \\
\hline $28 / 07 / 2018$ & 30 & Hidrocefalia no comunicante & $1 \mathrm{~h} 50 \mathrm{~min}$ \\
\hline
\end{tabular}

Tabla 2.

Acceso minipterional combinado con clinoidectomia y/o peeling de fosa media

\begin{tabular}{|l|l|l|l|c|}
\hline Fecha & Edad & \multicolumn{1}{|c|}{ Diagnóstico } & Minipterional combinado con & Tiempo quirúrgico \\
\hline $12 / 05 / 2017$ & 51 & HSA an paraclinoideo & Clinoidectomia & $6 \mathrm{~h} 51 \mathrm{~min}$ \\
\hline $01 / 06 / 2017$ & 46 & an paraclinoideo no roto & Clinoidectomia & $6 \mathrm{~h} \mathrm{24} \mathrm{min}$ \\
\hline $13 / 06 / 2017$ & 53 & HSA an paraclinoideo roto & Clinoidectomia & $6 \mathrm{~h} 00 \mathrm{~min}$ \\
\hline $22 / 06 / 2017$ & 52 & Meningioma seno cavernoso & Peeling + Clinoidectomia & $7 \mathrm{~h} 53 \mathrm{~min}$ \\
\hline $12 / 07 / 2017$ & 58 & Meningioma clinoidal & Clinoidectomia & $8 \mathrm{~h} 34 \mathrm{~min}$ \\
\hline $28 / 09 / 2017$ & 28 & Meningioma incisura tentorial & Peeling fosa media & $7 \mathrm{~h} 05 \mathrm{~min}$ \\
\hline $02 / 11 / 2017$ & 69 & an paraclinoideo no roto & Clinoidectomia & $5 \mathrm{~h} 54 \mathrm{~min}$ \\
\hline $08 / 11 / 2017$ & 48 & HSA an paraclinoideo & Clinoidectomia & $6 \mathrm{~h} 23 \mathrm{~min}$ \\
\hline $21 / 11 / 2017$ & 48 & an paraclinoideo no roto & Clinoidectomia & $5 \mathrm{~h} 49 \mathrm{~min}$ \\
\hline $29 / 11 / 2017$ & 56 & Meningioma tentorial & Peeling fosa media & $8 \mathrm{~h} 23 \mathrm{~min}$ \\
\hline $15 / 06 / 2018$ & 37 & HSA an art cerebral posterior & Clinoidectomia & $6 \mathrm{~h} 53 \mathrm{~min}$ \\
\hline
\end{tabular}

HSA: hemorragia subaracnoidea; an: aneurisma; acop: arteria comunicante posterior; acoa: arteria comunicante anterior.

\section{Tabla 3.}

Promedios de tiempos quirúrgicos

\begin{tabular}{|l|c|}
\hline Patología & Promedio del tiempo quirúrgico \\
\hline HSA por aneurisma no paradinoideo & $4 \mathrm{~h} 43 \mathrm{~min}$ \\
\hline HSA por aneurisma paradinoideo & $6 \mathrm{~h} 25 \mathrm{~min}$ \\
\hline Aneurisma no paradinoideo no roto* & $4 \mathrm{~h} 05 \mathrm{~min}$ \\
\hline Aneurisma paradinoideo no roto & $6 \mathrm{~h} 00 \mathrm{~min}$ \\
\hline Tumor de la base del cráneo & $7 \mathrm{~h} 59 \mathrm{~min}$ \\
\hline Hidrocefalia no comunicante & $1 \mathrm{~h} 58 \mathrm{~min}$ \\
\hline * Se consideró un aneurisma paradinoideo no roto, en el que no se realizó clinoidectomía extradural.
\end{tabular}

de cráneo resueltos por acceso minipterional combinado, seguido por los aneurismas paraclinoideos que requirieron combinar el acceso minipterional con clinoidectomia extradural. Por otro lado, el tiempo quirúrgico promedio menor, fue en relación a los casos de hidrocefalia no comunicante resueltos por acceso minipterional simple.

El $80,3 \%$ (45 casos) fueron aneurismas, $48 \%$ rotos (27 casos) y $32 \%$ (18 casos) no rotos; el $12,5 \%$ (7 casos) correspondieron a hidrocefalias no comunicantes y el $7 \%$ (4 casos) a tumores de la base de cráneo.

La mayoría de los pacientes con hemorragia subaracnoidea por aneurisma roto, ingresaron en buenas condiciones neurológicas, en WFNS I-III el $74 \%$. Ningún paciente fue operado en WFNS V. 


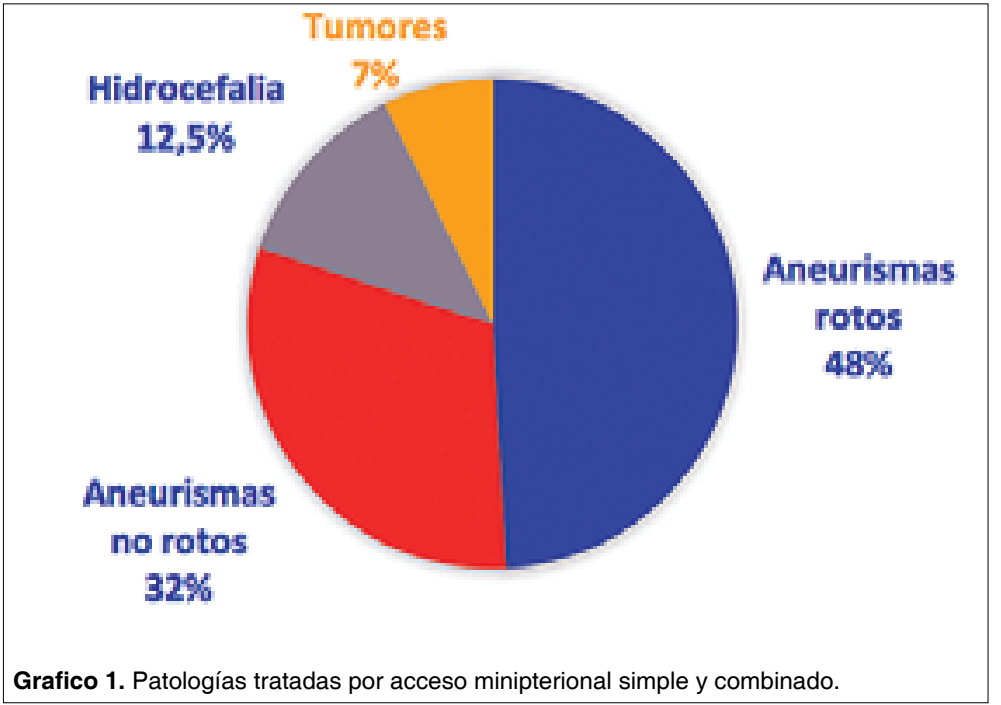

\section{Tabla 4}

Condición de ingreso de los pacientes con HSA

\begin{tabular}{|l|c|c|c|c|l|}
\hline WFNS & $\mathbf{1}$ & II & III & IV & V \\
\hline $\mathrm{N}$ & 8 & 4 & 8 & 7 & 0 \\
\hline$\%$ & $29,6 \%$ & $14,8 \%$ & $29,6 \%$ & $26 \%$ & $0 \%$ \\
\hline
\end{tabular}

\section{Tabla 5}

Escala de Fisher modificado en los pacientes con HSA

\begin{tabular}{|l|l|l|l|l|l|}
\hline Fisher modificado & $\mathbf{0}$ & $\mathbf{1}$ & II & III & IV \\
\hline $\mathrm{N}$ & 0 & 1 & 3 & 10 & 13 \\
\hline$\%$ & $0 \%$ & $3,7 \%$ & $11 \%$ & $37 \%$ & $48 \%$ \\
\hline
\end{tabular}

De los pacientes con HSA, el $85 \%$ (23 casos) tuvieron un Fisher alto (III-IV). En todos los pacientes con HSA, se realizó lavado cisternal, con apertura de la membrana de liliequist y lámina terminalis. En los pacientes con hidrocefalia se les instaló un drenaje ventricular externo, de manera transitoria, solo durante la cirugía. Dos pacientes presentaron hidrocefalia en el periodo post operatorio, requiriendo una deriva- tiva ventrículo peritoneal definitiva.

Los pacientes con aneurismas no rotos fueron asintomáticos, excepto dos pacientes con aneurisma del segmento comunicante, que presentaron paresia del III par completo, lo que se resolvió en ambos, posterior a la cirugía, a los 40 y 75 días respectivamente. En este grupo de pacientes no hubo complicaciones post operatorias.

De los 45 pacientes operados por aneu-

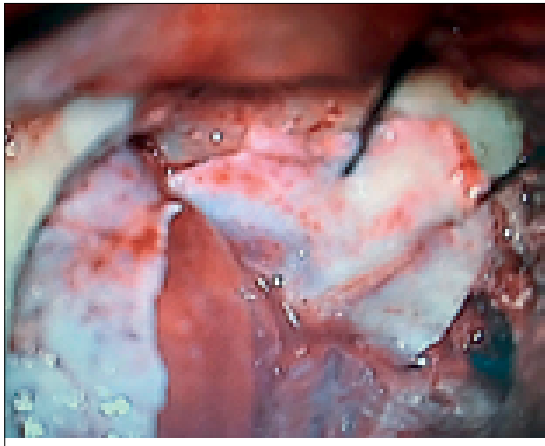

Figura 6. Durotomía arciforme. En un paciente con aneurisma silviano roto.

risma cerebral, 42 casos $(93,3 \%)$ tiene angiotac post operatorio, dos pacientes presentaron un remanente del aneurisma $(4,4 \%)$, un aneurisma paraclinoideo roto con un pequeño remanente medial y en un aneurisma silviano no roto, multilobulado, en el que se decidió dejar "ex profeso" un remanente ateromatoso que estaba en estrecha relación con una rama de la bifurcación silviana. La condición pre y post operatoria de los pacientes con tumores de la base del cráneo se describe en la Tabla 6. En el post operatorio no hubo déficit neurológico permanente agregado. Hubo dos casos, un meningioma clinoidal y otro del seno cavernoso, presentaron una paresia transitoria y parcial del III par craneal; otro caso, un meningioma tentorial, tuvo una paresia del IV par craneal también en forma transitoria. Hubo un caso de infección de herida operatoria, que requirió un aseo quirúrgico, sin mayores complicaciones y otro caso con fistula de líquido cefalorraquideo que requirió reparación de la misma.

En el control imageneológico post quirúrgico, por resonancia magnética, hubo evidencia de remanente tumoral solo en un caso, de un meningioma del seno cavernoso.

En todos los pacientes operados por hidrocefalia no comunicante, se logró

\section{Tabla 6.}

\section{Tumores de la base del cráneo. Condición pre y post quirúrgica}

\begin{tabular}{|l|l|l|}
\hline Patologías & Pre operatorio & Post operatorio \\
\hline Meningioma clinoidal & Sin déficit neurológico & Paresia del III par craneal transitoria \\
\hline Meningioma incisura tentorial & Sin déficit neurológico & Sin déficit neurológico. Fístula de LCR. \\
\hline Meningioma tentorial & Sin déficit neurológico & Paresia del IV par craneal transitoria \\
\hline Meningioma seno cavernoso derecho & Amaurosis derecha & Paresia del III par craneal derecho transitoria \\
\hline
\end{tabular}




\begin{tabular}{|c|c|c|}
\hline & Favorable $($ ERm $<3)$ & No favorable $(E R m>0=3)$ \\
\hline $\mathrm{N}$ & 49 & 7 \\
\hline$\%$ & $87,5 \%$ & $12,5 \%$ \\
\hline
\end{tabular}

la resolución de la hidrocefalia con la apertura de la lámina terminalis y no hubo complicaciones.

Del total de pacientes, 56 casos, en 49 $(87,5 \%)$ se obtuvo un resultado neurológico favorable $(\mathrm{ERm}<3)$. De los $7(12,5 \%)$ pacientes con resultados neurológicos no favorables $(\mathrm{ERm}>0$ $=3$ ), todos fueron hemorragias subaracnoideas aneurismáticas, 3 de ellos con WFNS IV, y todos cursaron con vasoespasmo severo, 5 de estos pacientes fallecieron (Tabla 7).

Todos los pacientes sometidos a cirugía electiva, aneurismas cerebrales no rotos, tumores de la base del cráneo e hidrocefalias no comunicantes, obtuvieron un resultado neurológico favorable, evaluados por la escala de Rankin modificado (ERm).

En cuanto a los resultados estéticos, tres pacientes presentaron atrofia del músculo temporal, no hubo complicaciones de cicatrización ni necrosis de colgajo. Un paciente presento paresia de la rama frontal del nervio facial.

\section{Caso clínico 1}

El paciente EOO, de 48 años, el 17 de junio de 2015 presentó una hemorragia subaracnoidea por un aneurisma de la arteria comunicante anterior roto, operado en esa fecha, por el mismo autor, por acceso pterional clásico (Figuras 7 y 8).

El paciente evoluciono favorablemente de la hemorragia subaracnoidea, posteriormente en forma electiva, se resolvieron los aneurismas no rotos, de bifurcación carotidea derecha y silviano izquierdo, el 14 y 22 de junio de 2018, respectivamente, por acceso minipterional (Figura 9 y 10).

\section{Caso clínico 2}

MJR, 56 años, paciente con antecedente de HTA, diabetes y sobrepeso, consulta por cefalea, se estudia con RM cerebral con gadolinio, que demos-

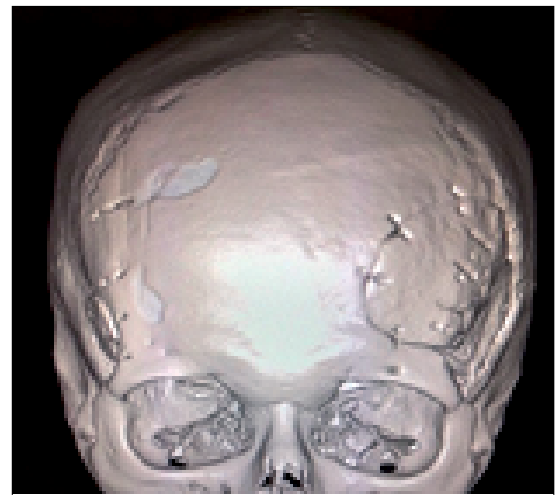

Figura 10. Tac 3d de superficie craneal, evidencia craneotomía pterional clásica izquierda y minipterional derecho.

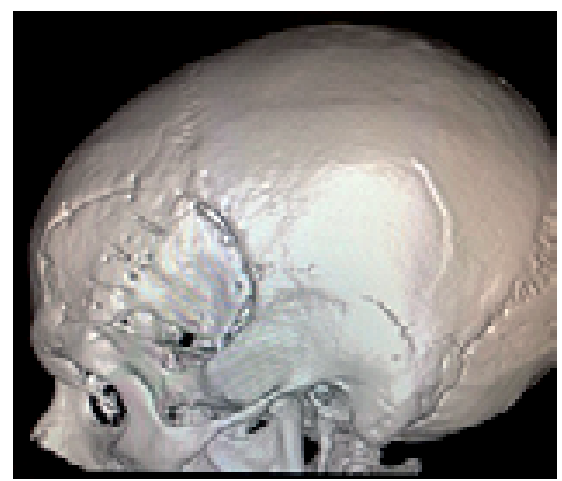

Figura 11. Craneotomía pterional clásica, utilizada para clipaje aneurisma acoa (año 2015).

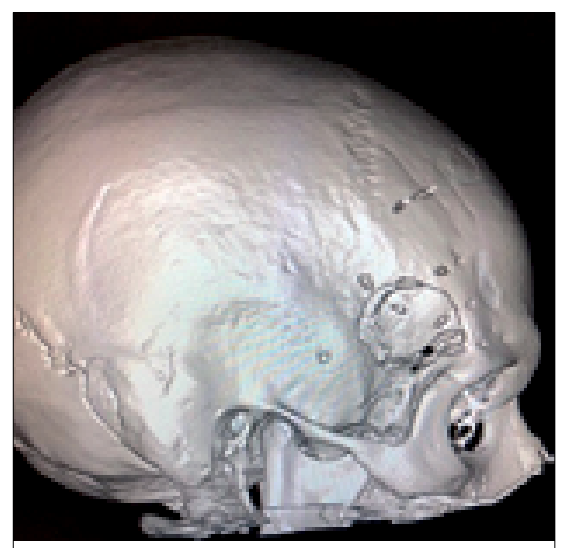

Figura 12. Craneotomía minipterional, utilizada para clipaje de aneurisma bifurcacion carotidea (año 2017).

tró meningioma tentorial (Figura 13). El 29/11/2017 se realizó la cirugía por acceso minipterional, con peeling de fosa media, logrando la resección completa (Figuras 14 y 15), la paciente evolucio- 


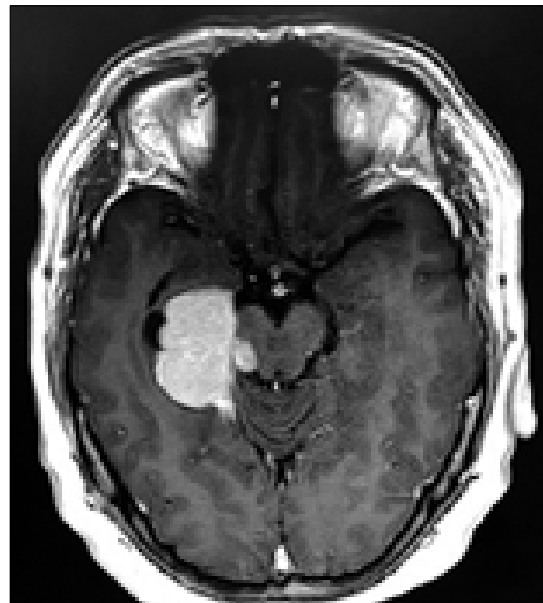

Figura 13. RM cerebral pre operatoria, meningioma tentorial derecho.

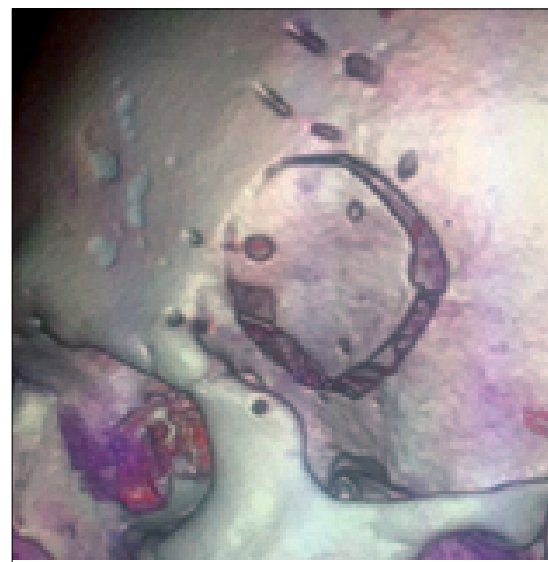

Figura 16. TC post operatorio, craneotomia, minipterional izquierda.

na favorablemente, solo con paresia transitoria del IV par craneal derecho.

\section{Caso clínico 3}

GEC paciente, de sexo femenino, de 69 años de edad, consulta por cefalea, se estudia con RM cerebral que evidenció un aneurisma paraclinoideo izquierdo, se completó el estudió con un angiotac cerebral (Figura 17). El 02 de noviembre de 2017 , se realiza la cirugía del clipaje del aneurisma por una craneotomía minipterional, combinada con clinoidectomia anterior extradural (Figuras 18, 19 y 20).

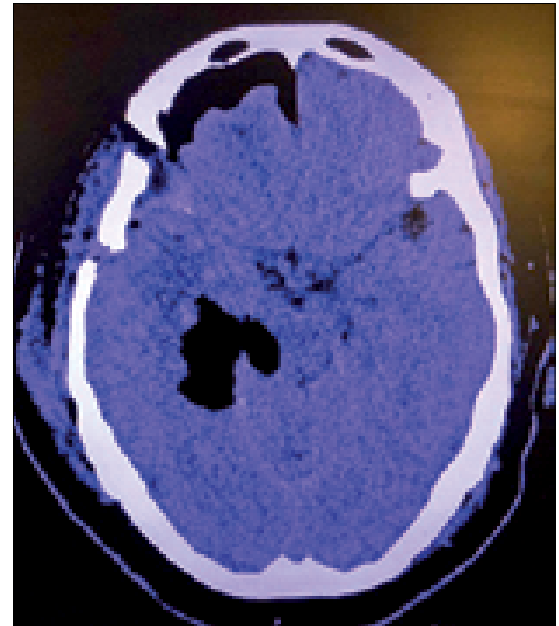

Figura 14. TC post operatorio inmediato, resección de la lesión, craneotomía minipterional.

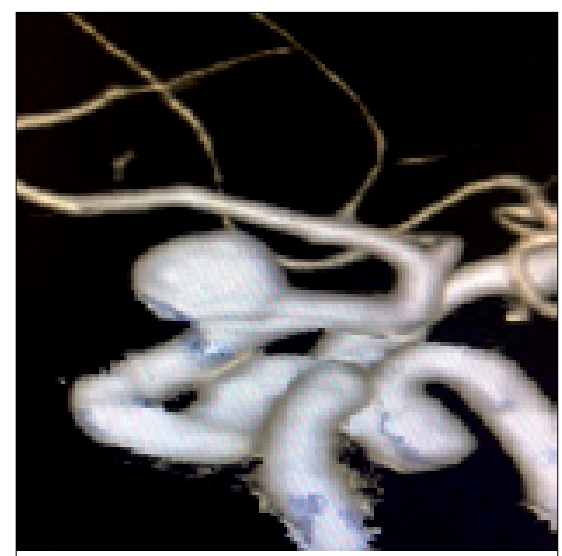

Figura 17. Angiotac cerebral, muestra aneurisma paraclinoideo izquierdo, carotido oftálmico.

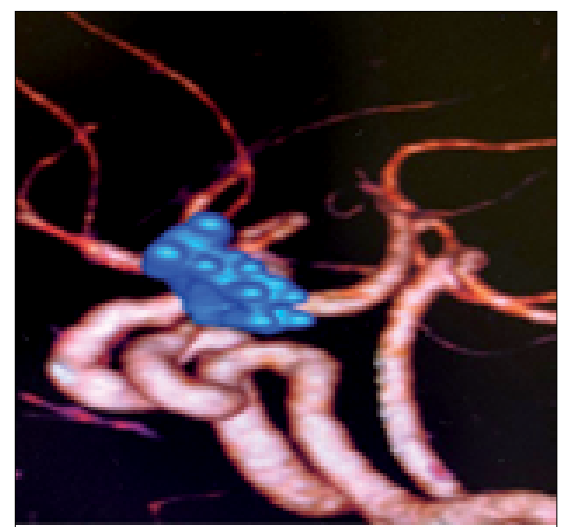

Figura 19. Angiotac post operatorio, exclusión de aneurisma y permeabilidad de $\mathrm{ACl}$.

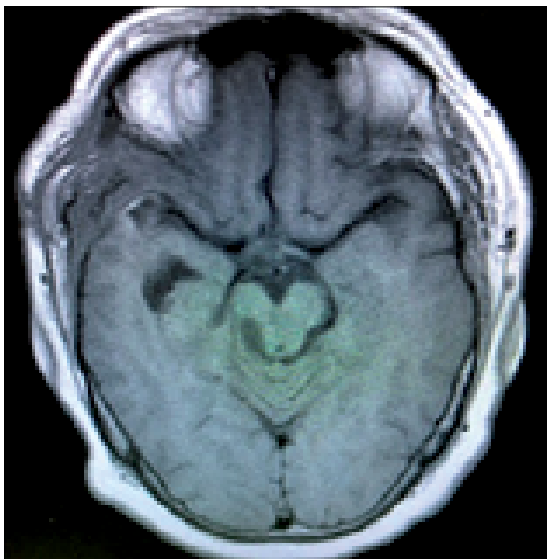

Figura 15. RM post operatoria, no se observa remanente tumoral.

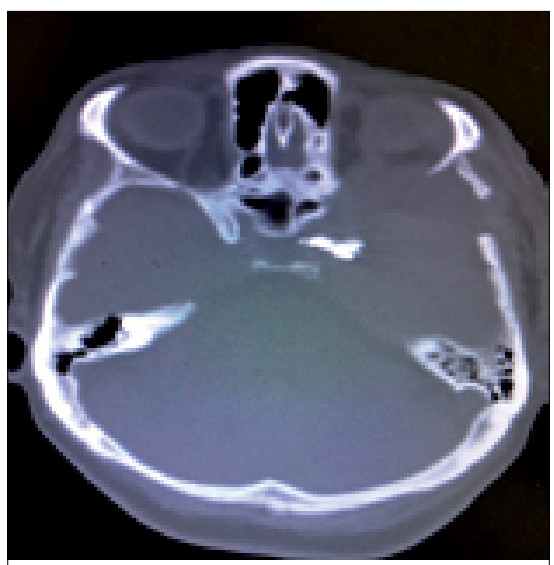

Figura 18. Tac post operatorio, muestra clinoidectomia anterior y clip en relación a esto.

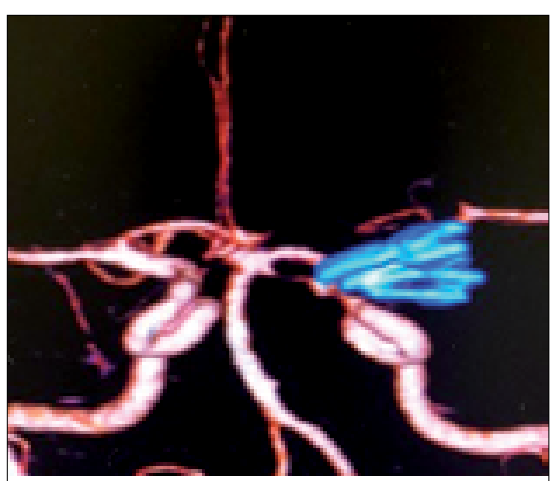

Figura 20. Tac con reconstrucción 3D de cráneo, craneotomía minipterional. 
La paciente evoluciona sin complicaciones, sin déficit neurológico, sin déficit visual. Siendo dada de alta al tercer día post operatorio.

\section{Discusión}

El acceso minipterional ha demostrado, tanto en esta serie clínica como en otras, ser tan versátil y eficiente como el acceso pterional clásico, además tiene la ventaja de disminuir los tiempos quirúrgicos, no tener las complicaciones en relación al seno frontal ${ }^{5}$, preservar siempre la arteria temporal superficial ${ }^{4}$, es menos frecuente la paresia de la rama frontal del nervio facial ${ }^{8}$ y tiene mejores resultados estéticos ${ }^{5}$. Por otro lado, en cuanto a los aneurismas rotos presentados en esta serie clínica, operados por una craneotomía minipterional, comparados con los tratados por el autor en el 2015, por craneotomía pterional clásica, no presentan limitaciones para la apertura de las cisternas de la base, el lavado cisternal, la disección y clipaje de aneurisma (Figuras 11 y 12). De manera que, a juicio del autor, la craneotomía minipterional, es el acceso de elección para lesiones del piso de la fosa anterior y media, así como para lesiones vasculares de la circulación anterior y aneurismas del top de la basi- lar. Al mismo tiempo se ha demostrado que, a través de una craneotomía mínima, se puede realizar cirugía compleja de la base del cráneo, combinándolo con un peeling de la fosa media, pudiendo llegar sin dificultad, al seno cavernoso y a la incisura tentorial; con la clinoidectomia anterior extradural, para aborda la región paraclinoidea y con la petrosectomía anterior se puede acceder al tercio superior del clivus. Sin embargo, una excepción son las lesiones que comprometen el centro del piso de la fosa anterior, con extensión más allá de la bifurcación carotidea contralateral, como grandes meningiomas del tubérculo selar o surco olfatorio o craneofaringiomas, en las cuales se considera la vía transbasal una mejor alternativa. Por otro lado, en casos seleccionados, se pudiese preferir la vía pterional clásica por sobre el minipterional, en HSA con grandes hematomas intraparenquimatosos, en patologías tumorales donde se planifique trabajar además de a través del valle silviano, por subfrontal la mayor parte del tiempo, logrando acceder de forma directa en el sentido antero-posterior y no de forma oblicua subfrontal como ofrece el minipterional. Es así como el autor, en un caso de meningioma del tubérculo selar, ha preferido el acceso pterional clásico por sobre el minipterional.

\section{Conclusión}

En esta serie clínica se presentó una variedad de patologías complejas, resueltas por la vía minipterional pura o combinada con peeling de fosa media y clinoidectomia anterior extradural, demostrando que se puede reproducir lo publicado por otros autores ${ }^{4,5,6,7,8}$, obteniendo resultados funcionales y estéticos favorables, en la mayoría de los pacientes, destacando que los resultados no favorables estuvieron asociados a complicaciones de la hemorragia subaracnoidea, no al acceso ni al acto quirúrgico. De manera que esta serie clínica, es un argumento más para continuar tratando pacientes por esta vía, la craneotomía minipterional, siendo un acceso reproducible, con ventajas sobre el pterional clásico y otros accesos keyhole $^{5,12}$ en la mayoría de las patologías del piso anterior y fosa media, además combinado con clinoidectomia anterior y/o peeling de fosa media, se pueden resolver patologías que antes requerían accesos amplios, como el transcigomático u orbitocigomático, que ofrecen una mayor morbilidad.

\section{Recibido: 31 de diciembre de 2018 Aceptado: 15 de enero de 2019}

\section{Referencias}

1. Yasargil MG, Fox JL. The microsurgical approach to intracranial aneurysms. Surg Neurol. 1975;3(1):7-14.

2. Yasargil MG, Antic J, et al. Microsurgical pterional approach to aneurysms of the basilar bifurcation. Surg Neurol, $1976 ; 83-91$.

3. Zabramski JM, Kiris T, Sankhla SK, et al. Orbitozygomatic craniotomy. Technical note. J Neurosurg 1998;89:336-41.

4. Jun-Young A, Superficial Temporal Artery-Sparing Mini-Pterional Approach for Cerebral Aneurysm Surgery. J Korean Neurosurg Soc. 2017 Jan 1;60(1):8-14.

5. Welling LC, Prospective randomized study comparing clinical, functional, and aesthetic results of minipterional and classic pterional craniotomies. J Neurosurg. 2015 May;122(5):1012-9.

6. Chaulohi N, Surgical treatment of ruptured anterior circulation aneurysms: comparison of pterional and supraorbital keyhole approaches. Neurosurgery. 2013 Mar;72(3):437-41.

7. Nathal E, Gómez-Amador JL: Anatomic and surgical basis of the sphenoid ridge keyhole approach for cerebral aneurysms. Neurosurgery 56:178-185, 2005.

8. Figueiredo E, Deshmukh P, Nakaji P, Crusius M, Crawford N, Spetzler R, Preul M. The minipterional craniotomy: technical description and anatomic assessment. J Neurosurg. 2007;61:256-265.

9. Hakuba A, Nishimura S, Shirakata S, Tsukamoto M. Surgical Approaches to the Cavernous Sinus. Neurol med chir. Tokio. 1982; 22:295-308.

10. Dolenc V, Direct microsurgical repair of intracavernous vascular lesions. J Neurosurg 1983;58:824-83.

11. Dolenc V, A combined epi and subdural direct approach to carotid-ophthalmic artery aneurysms. J Neurosurg. 1985;62:667-672.

12. Rychen J, Minimally Invasive Alternative Approaches to Pterional Craniotomy: A Systematic Review of the Literature. World Neurosurg 2018 May;113:163-179.

\section{Correspondencia a:}

\section{Dr. Iván Perales Cabezas}

Neurocirujano Vascular y de la Base del Cráneo. Hospital San Pablo y Clínica Elqui. Región de Coquimbo, Chile.

Loteo encomenderos Arnoldo Vasquez, 39-A. La Serena. Chile.

Telefono: +56962291283

ivanperalescabezas@gmail.com 